\title{
Disruption of TWIST1-RELA binding by mutation and competitive inhibition to validate the TWIST1 WR domain as a therapeutic target
}

Cai M. Roberts ${ }^{1,3}$, Sophia A. Shahin ${ }^{1}$, Joana Loeza ${ }^{2,4}$, Thanh H. Dellinger', John C. Williams ${ }^{1}$ and Carlotta A. Glackin ${ }^{1 *}$

\begin{abstract}
Background: Most cancer deaths result from tumor cells that have metastasized beyond their tissue of origin, or have developed drug resistance. Across many cancer types, patients with advanced stage disease would benefit from a novel therapy preventing or reversing these changes. To this end, we have investigated the unique WR domain of the transcription factor TWIST1, which has been shown to play a role in driving metastasis and drug resistance.
\end{abstract}

Methods: In this study, we identified evolutionarily well-conserved residues within the TWIST1 WR domain and used alanine substitution to determine their role in WR domain-mediated protein binding. Co-immunoprecipitation was used to assay binding affinity between TWIST1 and the NFKB subunit p65 (RELA). Biological activity of this complex was assayed using a dual luciferase assay system in which firefly luciferase was driven by the interleukin-8 (IL-8) promoter, which is upregulated by the TWIST1-RELA complex. Finally, in order to inhibit the TWIST1-RELA interaction, we created a fusion protein comprising GFP and the WR domain. Cell fractionation and proteasome inhibition experiments were utilized to elucidate the mechanism of action of the GFP-WR fusion.

Results: We found that the central residues of the WR domain (W190, R191, E193) were important for TWIST1 binding to RELA, and for increased activation of the IL-8 promoter. We also found that the C-terminal 245 residues of RELA are important for TWIST1 binding and IL-8 promoter activation. Finally, we found the GFP-WR fusion protein antagonized TWIST1-RELA binding and downstream signaling. Co-expression of GFP-WR with TWIST1 and RELA led to proteasomal degradation of TWIST1, which could be inhibited by MG132 treatment.

Conclusions: These data provide evidence that mutation or inhibition of the WR domain reduces TWIST1 activity, and may represent a potential therapeutic modality.

Keywords: TWIST1, RELA, WR domain, Protein-protein interactions, Protein degradation

\footnotetext{
* Correspondence: cglackin@coh.org

'City of Hope, 1500 E Duarte Rd, Duarte, CA 91010, USA

Full list of author information is available at the end of the article
} 


\section{Background}

The majority of cancer deaths are the result of tumor cells metastasizing beyond their original niche [1]. Disseminated disease is difficult to resect and may be genetically different to the primary tumor [2]. Moreover, acquisition of drug resistance further complicates effective therapeutic approaches. In ovarian cancer in particular, late stage at discovery and drug resistance are major challenges [3, 4], resulting in five year survival rates of approximately $25 \%$ $[3,5]$. Thus, in ovarian and other cancers, a novel therapeutic strategy capable of addressing both metastasis and drug resistance is urgently needed.

A promising target for such an approach is the transcription factor TWIST1. TWIST1 expression and activity is essential in early development but is not retained in adults. However, many cancers reactivate TWIST1 expression [6-8]. In both the developmental and cancer contexts, TWIST1 drives epithelial to mesenchymal transition (EMT), in which cells alter their phenotype, including elongated morphology and expression of cell surface proteins, to facilitate migration and invasion [7]. Enhanced cellular motility in turn gives rise to mesodermal tissues in embryogenesis and to metastases in cancer [7, 8]. Furthermore, TWIST1 has been implicated in number of pro-progression phenotypes in cancers, including angiogenesis [9], increased cancer cell stemness [10-13], and cell survival signaling [14, 15] (Fig. 1a).

TWIST1 has well-characterized transcription factor activity; its dimerization partners and binding site within target promoters have been elucidated previously [16, 17]. Recently, more studies have focused on the Twist box or WR domain, comprised of the C-terminal twenty amino acids of the protein (Fig. 1b). The TWIST1 gene is well conserved evolutionarily, but this is especially true for the WR domain; $100 \%$ homology is preserved from human to Xenopus (Fig. 1c). We have previously shown that the WR domain mediates a binding interaction between TWIST1 and the NF- $\mathrm{kB}$ subunit RELA, and that this interaction leads to transcriptional upregulation of the inflammatory cytokine interleukin 8 (IL-8) in a manner independent of TWIST1-DNA binding [18]. Furthermore, Piccinin et al. demonstrated a binding interaction between the WR domain and the C-terminus of the tumor suppressor p53, which led to p53 degradation [19]. Recently, it was revealed that the WR domain can also bind to the WR domain of a nearby TWIST1-E47 heterodimer, thereby creating higher order complexes required for proper transcriptional regulation of target genes [17]. This finding may explain the finding that altered TWIST1-mediated transcription of Hoxa9 was responsible for the inability of prostate cancer cells expressing WR-truncated alleles of TWIST1 to metastasize in an in vivo model system [20].

Given its importance in mediating not only proteinprotein interactions, but also the DNA binding activity of TWIST1, we hypothesize that the WR domain is a potential target to block TWIST1 functions associated with cancer. To test this hypothesis, we sought specific residues mediating the interaction with RELA and tested mutants lacking these residues using our previously validated model system [18]. We further demonstrate that a WR domain mimetic can abrogate TWIST1 activity in vitro, providing further evidence that blocking this interaction and inhibiting TWIST1 expression could be an effective cancer therapeutic strategy.

\section{Methods}

\section{Cell culture}

HEK-293 cells were grown in McCoy's 5A medium supplemented with $10 \%$ fetal bovine serum (FBS) and $1 \%$ penicillin/streptomycin (P/S). Ovcar4 cells were grown in RPMI medium with $10 \% \mathrm{FBS}$ and $1 \% \mathrm{P} / \mathrm{S}$. All cells were maintained at $37{ }^{\circ} \mathrm{C}$ and $90 \%$ humidity in a tissue culture incubator with $5 \% \mathrm{CO}_{2}$ atmosphere. Cells were passaged every 2-4 days as they became confluent, using $0.25 \%$ trypsin. Where indicated, cells were transfected using $5 \mu \mathrm{L}$ per well Lipofectamine 2000 (Life Technologies, Carlsbad, CA) in a total of $2 \mathrm{~mL}$ per well of OptiMEM low serum medium (Life Technologies). Cycloheximide (CHX) was obtained from Sigma Aldrich (St. Louis, MO) and used at a dose of $20 \mu \mathrm{g} / \mathrm{ml}$. For CHX studies, cells were transfected using XtremeGene 9, also from Sigma Aldrich. For proteasome inhibition studies, MG132 was added to HEK-293 cells in normal medium four hours after transfection and left on overnight. A dose of $5 \mu \mathrm{M}$ was used for fractionated western studies and $1 \mu \mathrm{M}$ was used for luciferase assays.

\section{Site directed mutagenesis}

The cloning of TWIST1 into the pcDNA4-MycHis vector has been described previously [18]. The wild type RELA gene was also cloned into pcDNA4-MycHis, including a stop codon at the C-terminus to prevent translation of the Myc-His tag. TWIST1 retained the tag. Amino acid substitution and truncation mutations were introduced using the QuikChange II site directed mutagenesis kit (Agilent, Santa Clara, CA) according to the manufacturer's instructions and following their recommendations for primer design. Silent mutations were introduced in tandem with the desired mutations in order to create or eliminate restriction sites to facilitate screening for mutants. All mutations were confirmed by Sanger sequencing by the City of Hope Integrative Genomics Core.

\section{GFP fusion protein}

In order to create a competitive inhibitor for TWIST1RELA binding, the WR domain from TWIST1 was fused to eGFP. Briefly, PCR was used to amplify the final 63 nucleotides of the TWIST1 gene (including stop codon) and add 5' XhoI and 3' BamHI restriction sites. The 


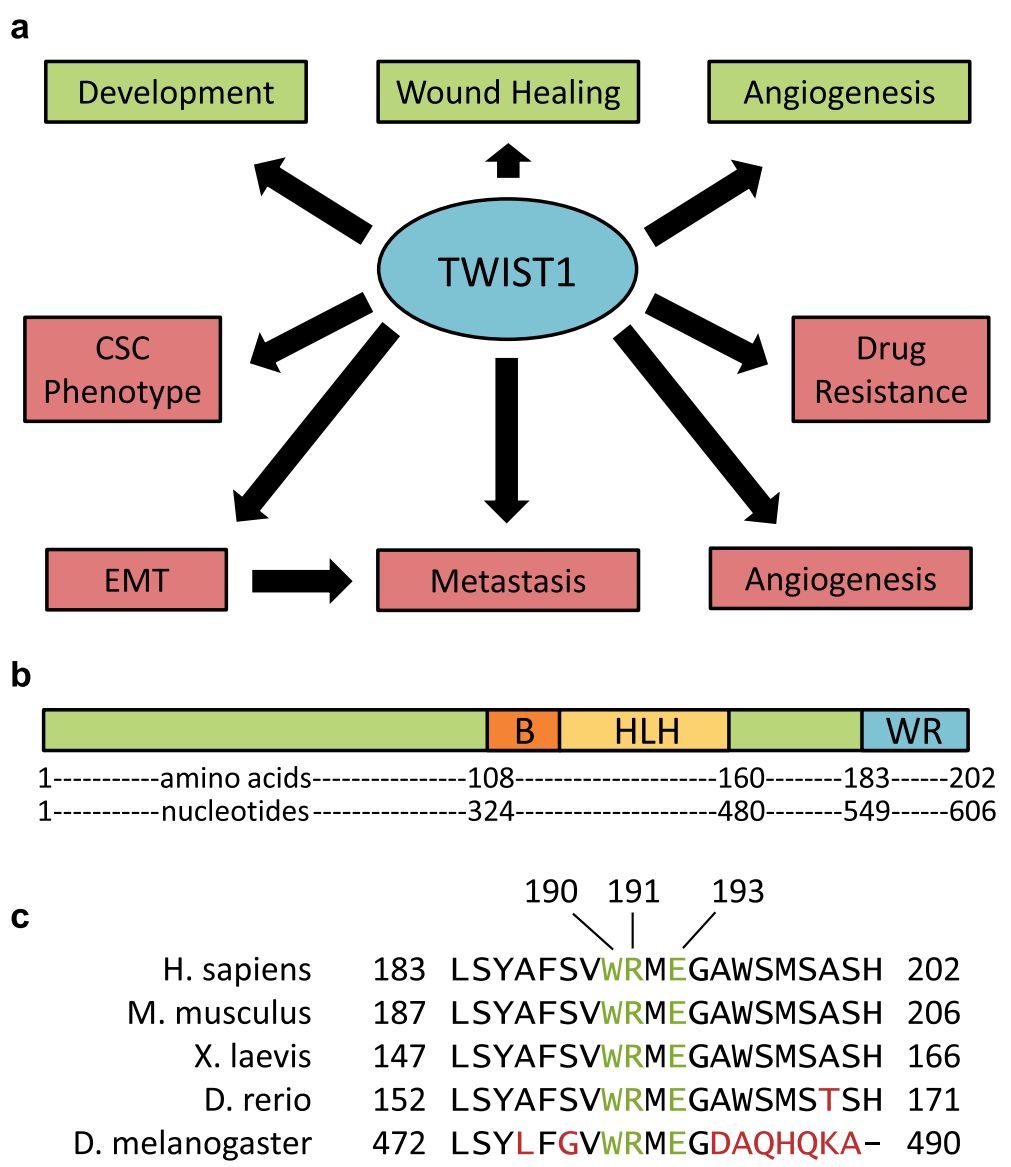

Fig. 1 TWIST1 is a highly conserved bHLH class transcription factor with multiple functions. a TWIST1 functions in normal development and in small populations of adult stem cells, where it assists in wound healing. When reactivated in cancers, TWIST1 activates a transcriptional and protein binding program giving rise to EMT, and thus to metastases. Many studies have also linked re-expression of TWIST1 to the acquisition of drug resistance and an increase in stemness. Functions in normal tissue are shown in green; in cancer, in red. $\mathbf{b}$ Human TWIST1 protein is 202 amino acids in length, with the N-terminal half of the protein being largely disodered The C-terminal half consists of the basic DNA binding domain (orange), helix-loop-helix dimerization domain (yellow), and the Twist box or WR domain (blue), which has been shown to be a transactivation domain. $\mathbf{c}$ The WR domain is especially well conserved throughout evolution, with 100\% identity between human, mouse, and frog. The central residues appearing in green are present in all organisms listed, including Drosophila, and for this reason, residues were selected for mutation

PCR fragment and the pEGFP-C3 vector were subjected to XhoI-BamHI double digest (New England BioLabs, Ipswich, MA) and the two fragments ligated together. GFP lacking the WR domain was used as a control, and includes 21 residues at the $\mathrm{C}$-terminus encoded by the multiple cloning site of the vector. As a result, the molecular weights of the two GFP proteins are indistinguishable on a western blot. To achieve equal expression of GFP-WR compared to unmodified GFP, it was necessary to transfect cells with three-fold more GFP-WR plasmid versus GFP. A one to one ratio was sufficient for CoIP illustrated in Fig. 4C. For all GFP-WR experiments, 4x refers to GFP-WR only, $3 x$ to a $3: 1$ ratio of GFP-WR to GFP, $2 \mathrm{x}$ to equal amounts of both, $1 \mathrm{x}$ to a $1: 3$ ratio of GFP-WR to GFP, and 0 to GFP only.

\section{Co-Immunoprecipitation}

HEK-293 cells were plated at 500,000 cells per well, in $2 \mathrm{~mL}$ normal medium, in a 6 well plate and allowed to adhere. The next day, medium was replaced with OptiMEM low serum medium (Life Technologies). Cells were transfected with various alleles of TWIST1, RELA, and GFP using Lipofectamine 2000 (Life Technologies). The following day, cells were detached using trypsin, washed with PBS, and pelleted. Cell pellets were lysed in RIPA buffer, and protein concentration was determined by BCA Protein Assay (Thermo Fisher, Waltham, MA). 50-100 $\mu$ g total protein (equal between conditions) was pre-cleared by incubating with $1 \mu \mathrm{g}$ normal rabbit IgG (Santa Cruz Biotechnology, Dallas, TX) and 20-30 $\mu \mathrm{L}$ Protein A/G Agarose beads (Santa Cruz Biotechnology, sc-2003) on a rocker at $4{ }^{\circ} \mathrm{C}$ for $1 \mathrm{~h}$. Water was added to 
equalize volumes across conditions. Beads were centrifuged for $1 \mathrm{~min}$ at 3,000 rpm, and equal volumes of supernatant from each condition were transferred to new tubes, and incubated with $1 \mu \mathrm{g}$ rabbit anti-RELA (Santa Cruz Biotechnology sc-109) or rabbit anti-GFP (Santa Cruz Biotechnology, sc-8334) antibodies on a rocker at $4{ }^{\circ} \mathrm{C}$. After $1 \mathrm{~h}, 20-30 \mu \mathrm{L}$ (equal between conditions) Protein A/G Agarose beads were added to each tube, and tubes were returned to the rocker at $4{ }^{\circ} \mathrm{C}$ overnight. The following day, unbound protein was removed and beads were washed five times with $1 \mathrm{~mL}$ PBS. Beads were boiled in $20 \mu \mathrm{L} 2 \mathrm{x}$ loading dye to release bound protein. Equal masses of input and equal volumes of immunoprecipitated protein were used for western blotting.

\section{Cell fractionation}

HEK-293 cells were plated and transfected as described for co-immunoprecipitation above. The following day, cells were detatched using trypsin, washed with PBS, and pelleted. Pellets were resuspended in $100 \mu \mathrm{L}$ hypotonic buffer $(10 \mathrm{mM}$ HEPES, $10 \mathrm{mM} \mathrm{KCl}, 0.1 \mathrm{mM}$ EDTA, $1 \mathrm{mM} \mathrm{Na} \mathrm{VO}_{4}, 1.25 \mathrm{mM} \mathrm{NaF}, 0.4 \%$ IGEPAL, $0.5 \mathrm{mM} \mathrm{DTT}$ ) in the presence of protease inhibitor (Thermo Fisher, Waltham, MA). Cells were left on ice 15 min to swell, and then lysed by addition of NP-40 to a final concentration of $0.1 \%$. Nuclei were separated from cytoplasmic lysate by centrifugation (3000 rpm, $10 \mathrm{~min}, 4^{\circ} \mathrm{C}$ ) and washed once in hypotonic buffer without NP-40. Nuclei were then resuspended in $50 \mu \mathrm{L}$ high salt buffer (20 mM HEPES, $400 \mathrm{mM} \mathrm{NaCl}, 1 \mathrm{mM}$ EDTA, $10 \%$ glycerol, $1 \mathrm{mM} \mathrm{Na} \mathrm{VO}_{4}, 1.25 \mathrm{mM} \mathrm{NaF}, 0.5 \mathrm{mM}$ DTT) plus protease inhibitor. Vials were shaken for $2 \mathrm{~h}$ at $250 \mathrm{rpm}$ at $4{ }^{\circ} \mathrm{C}$, and then centrifuged $(5 \mathrm{~min}$, $\left.14,800 \mathrm{rpm}, 4{ }^{\circ} \mathrm{C}\right) . \mathrm{NaCl}$ concentration was adjusted to $137 \mathrm{mM}$ by addition of water prior to western blotting.

\section{Cycloheximide study}

HEK-293 cells were plated at 150,000 or 250,000 per well in 12 well plates and allowed to adhere overnight. The following day, cells were transfected as described for the above procedures. On the third day, non-treated cells were harvested and cycloheximide was added to the remaining wells. Remaining treated cells were harvested at the indicated time points and used for western blotting.

\section{Western Blotting}

Protein was run on $10 \%$ resolving polyacrylamide gels and transferred to PVDF membrane. Membranes were rinsed with PBS and blocked in 5-10\% milk, $1 \mathrm{~h}$ at room temperature or overnight at $4{ }^{\circ} \mathrm{C}$. Membranes were then incubated with mouse primary antibody in milk with Tween-20 (Ab Buffer) for $1 \mathrm{~h}$ at room temperature or overnight at $4{ }^{\circ} \mathrm{C}$, and washed in PBS with $0.1 \%$ Tween20 (PBST). Membranes were then incubated with anti- mouse secondary antibody in Ab Buffer for $1 \mathrm{~h}$ at room temperature, followed by an additional five PBST washes. Primary antibodies were: TWIST1, TWIST 2c1a (Santa Cruz Biotechnology sc-81417) 1:250-1:500; for RELA, NF-кB p65 F-6 (Santa Cruz Biotechnology sc8008) 1:250-1:500; for GFP, GFP B-2 (Santa Cruz Biotechnology sc-9996) 1:1000; for actin, Sigma Aldrich A1978 or 2066. Secondary antibodies were HRP conjugated anti-mouse and anti-rabbit. Protein was detected using Blue Devil Film (Genesee) and ECL Plus (Thermo Fisher) or digital imaging. Quantitation of digital images was performed using the accompanying software from Syngene (Frederick, MD) or Carestream MI (Woodbridge, $\mathrm{CT}$ ).

\section{Luciferase assay}

Ovcar3 and Ovcar4 cells were plated at 50,000 or 75,000 cells per well, in $500 \mu \mathrm{L}$ RPMI, in a 24 well plate and allowed to adhere overnight. Ovcar4 cells were used for all luciferase assays except for that shown in Additional file 1: Figure S1. The following day, cells were switched to OptiMEM medium and transfected using Lipofectamine 2000 at $2 \mu \mathrm{L}$ per well. Plasmids were: TWIST1 in pcDNA4, RELA in pcDNA4, Renilla luciferase, and firefly luciferase $(F F l u c)$ in pGL3. FFluc was under the control of the IL-8 promoter; construction of this vector has been described previously [18]. Empty pGL3 lacking a promoter was used as a negative control for FFluc expression. Each condition was tested in triplicate. The day after transfection, luciferase expression was quantified using the Dual Luciferase Assay kit (Promega, Madison, WI) according to the manufacturer instructions.

\section{Confocal microscopy}

HEK-293 cells were plated at 500,000 per well in glass bottom $35 \mathrm{~mm}$ cell culture dishes, and the next day were transfected with TWIST1, RELA, and GFP or GFP-WR as described above. After a further $24 \mathrm{~h}$, cells were rinsed with PBS and stained for 15 min with DAPI. DAPI was then replaced with PBS. Images were captured using a Zeiss LSM700 Confocal Microscope and ZEN 2012 microscopy software (Zeiss AG, Oberkochen, Germany).

\section{Data analysis and statistics}

Western blots were quantified using GeneTools software. Data were graphed and analyzed in Microsoft Excel and GraphPad Prism 6, respectively. Luciferase assays were analyzed using one-way ANOVA with correction for multiple comparisons. For assay testing RELA mutants, all conditions were compared to all others. For assays testing TWIST1 mutants and GFP-WR inhibitor, positive control condition was compared to all others. Positive control conditions are indicated in each relevant 
figure. Cell counts were averages of four counts, and prior testing has demonstrated that the count is accurate to within $13 \%$. All error bars represent standard deviation. *, $p<.05 ;{ }^{* * *}, p<.01 ;{ }^{* * *}, p<.001 ;^{* * * * *}, p<.0001$ throughout.

\section{Results \\ Single amino acid changes in the WR domain disrupt TWIST1-RELA binding}

Site-directed mutagenesis was used to generate mutations in the WR domain of TWIST1. On the basis of their high evolutionary conservation (Fig. 1c), we selected W190, R191, and E193 for mutation to alanine (W190A, R191A, E193A alleles, respectively). The $\triangle \mathrm{WR}$ allele, in which all twenty amino acids of the WR domain have been deleted, was created previously as described elsewhere [18]. Mutants were screened by restriction digestion and confirmed by sequencing (data not shown). All alleles are shown schematically in Fig. 2a. In order to determine the contribution of individual amino acids in the WR domain to TWIST1-RELA binding, we transiently expressed RELA and all TWIST1 alleles in HEK293 cells and performed co-immunoprecipitation (CoIP). Following RELA pulldown, western blotting showed that as demonstrated previously, truncation of the entire WR domain reduced TWIST1 co-precipitation to basal levels. W190A, R191A, and E193A mutations reduced TWIST1 co-precipitation by 50-60\%. A triple mutant with W190A, R191A, and E193A mutations also reduced RELA binding by 60\%, with less variability (Fig. 2b-c).

\section{Ability of mutant TWIST1 to drive expression of IL-8 is reduced}

We have previously established that formation of a TWIST1-RELA complex upregulates IL- 8 expression by 2-2.5 fold over RELA alone, and that prevention of binding by truncating TWIST1 returns IL- 8 expression to basal levels [18]. In order to determine the effect of W190A, R191A, and E193A mutations on IL-8 promoter activity, we performed a dual luciferase assay in which firefly luciferase (FFluc) was under the control of the IL8 promoter. As expected, exogenous expression of RELA in Ovcar4 cells gave rise to a basal level of IL-8 driven FFluc, which was increased by co-expression of, and thus binding with, TWIST1 (Fig. 2d). Mirroring the phenotypes seen in our CoIP experiments above, W190A, R191A, and E193A mutations reduced expression of FFluc by $50 \%$, and the triple mutant reduced FFluc expression a further $10-20 \%$ compared to the single point mutants (Fig. 2d). Similar results were obtained using the cell line Ovcar3 (Additional file 1: Figure S1), but a better range of IL-8 promoter induction was achieved in Ovcar4, and this line was selected for all subsequent functional assays.
RELA C-terminus is required for TWIST1 binding

While we have shown that the TWIST1 C-terminus is required for complex formation with RELA, the required residues of RELA remained unknown. In order to locate this site, we created a truncation mutant of RELA, $\Delta 307$ (Fig. 3a). Site directed mutagenesis was employed to insert a stop codon directly following the coding sequence for the REL homology domain, a wellconserved domain that has been structurally characterized [21]. CoIP of RELA revealed that truncation of RELA reduced co-precipitation of TWIST1 by approximately $90 \%$ (Fig. 3b-c). Truncating both proteins resulted in a greater loss of binding; under these conditions, only $1.86 \%$ of wild type levels of TWIST1 was detectable following CoIP (Fig. 3b).

RELA C-terminus is required for IL-8 activation, independent of TWIST1 mutation status

In order to verify that loss of binding between RELA $\Delta 307$ and TWIST1 impacted IL-8 expression, we again utilized a dual luciferase assay. As expected, RELA truncation was able to reduce FFluc expression (Fig. 3d). However, this phenotype was independent of TWIST1; in the absence of TWIST1, RELA $\triangle 307$ produced only $30 \%$ of wild-type IL-8 promoter activity. TWIST1 expression upregulated IL-8-driven FFluc approximately two-fold, regardless of RELA status. As seen previously, co-expression of triple mutant TWIST1 with RELA led to an intermediate phenotype, for both WT and $\triangle 307$ alleles of RELA (Fig. 3d). Thus, we conclude that the domains required for both IL- 8 transactivation and complexing with TWIST1 are contained within the relatively uncharacterized C-terminus of RELA.

\section{Creation of a GFP-WR domain fusion protein}

Given the demonstrated role for the WR domain in RELA binding, as well as in the transcription factor activity of TWIST1 $[17,20]$, we propose that this domain is an attractive target for therapeutic intervention. To test whether the WR domain could act as a competitive inhibitor of TWIST1-RELA binding, the WR domain was fused to GFP in the pEGFP-C3 vector (Fig. 4a). Empty pEGFP-C3 encodes GFP followed by 21 residues encoded by the multiple cloning site. We therefore used this vector as a negative control, since its protein product would be of the same size as GFP-WR (Fig. 4a). Both forms of GFP could be expressed to similar degrees in HEK293 cells (Fig. 4b).

\section{GFP-WR fusion protein reduces TWIST1-RELA binding and IL-8 activation}

To determine the effect of GFP-WR on TWIST1-RELA binding, we performed CoIP analyses. Total GFP expression in transfected cells was held constant across all 


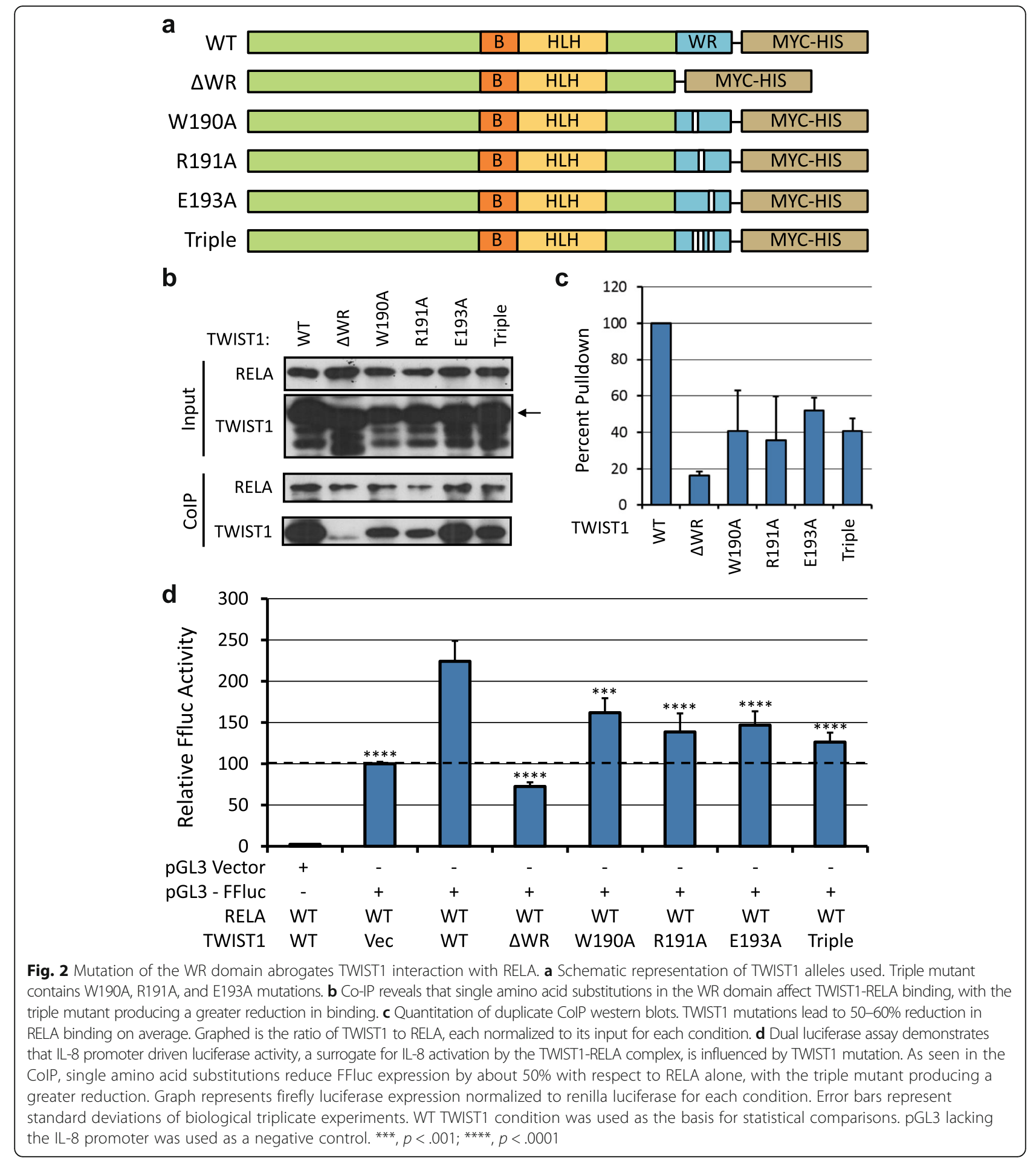

conditions by supplementing GFP-WR with control GFP. RELA pulldown revealed that levels of TWIST1 co-precipitated were reduced in a dose dependent fashion with increasing GFP-WR expression (Fig. 4c). GFP pulldown revealed that TWIST1 was co-precipitated in a dose-dependent manner with increasing GFP-WR expression (Fig. 4d). These findings suggest that GFP-WR is interacting with TWIST1 via WR-WR binding, an interaction illustrated by recent studies of higher order TWIST1 complexes [17]. In order to determine whether GFP-WR-mediated inhibition of TWIST1-RELA binding impacted downstream signaling, we again employed a dual luciferase assay to quantify IL-8 promoter activity. As expected, GFP-WR expression led to a dose- 
a

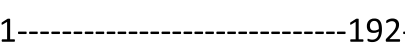

306 551

WT $\square$ REL HOMOLOGY DOMAIN $\triangle 3 0 7 \longdiv { \text { REL HOMOLOGY DOMAIN } }$

b
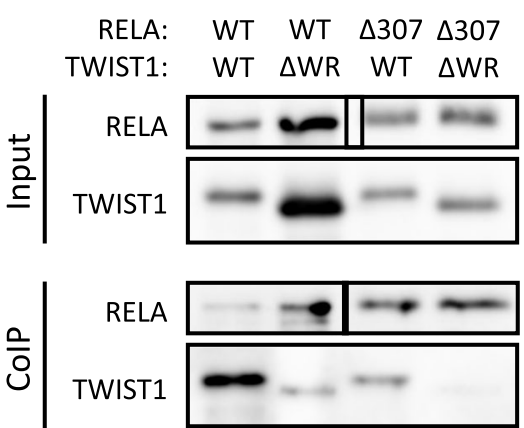

Normalized $\begin{array}{lllll}\text { TWIST1/RELA: } & 100 & 2.49 & 8.17 & 1.86\end{array}$
C

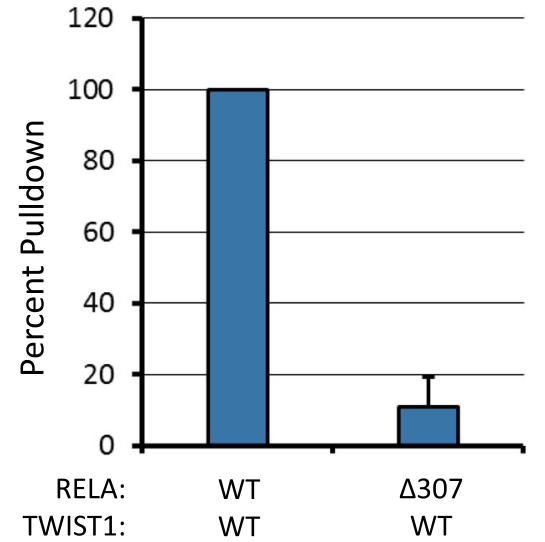

d

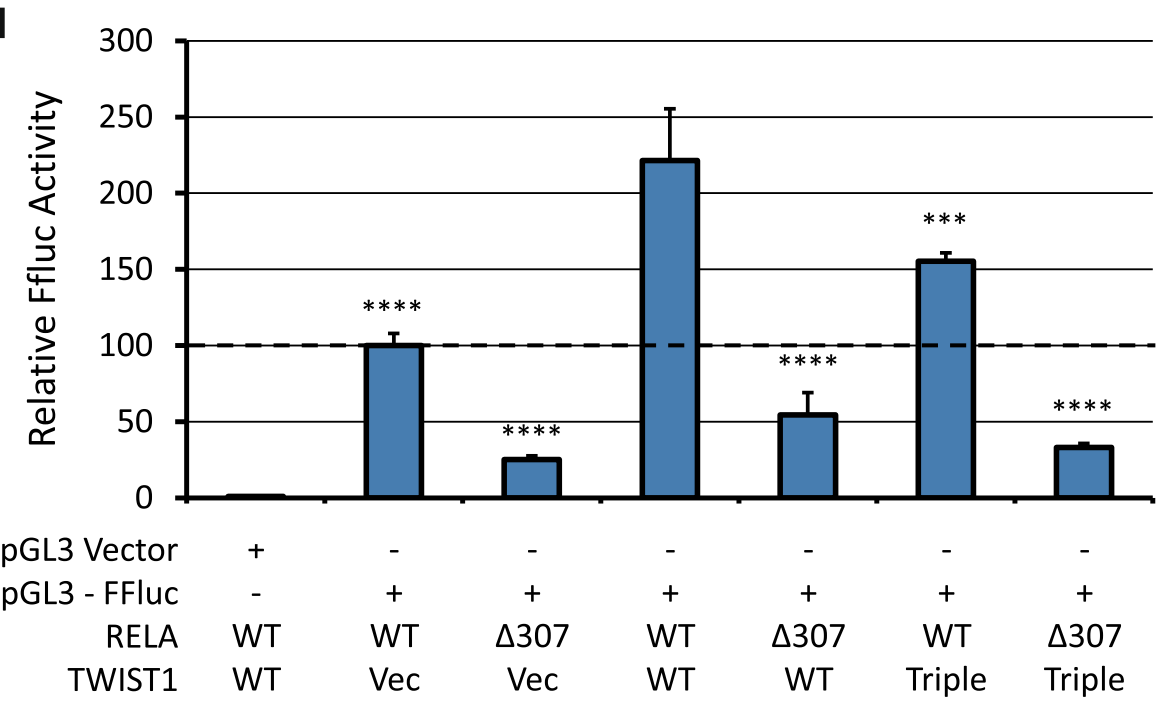

Fig. 3 Truncation of RELA reveals TWIST1 binding domain is also required for IL-8 regulatory activity. a Schematic representation of RELA alleles. b CoIP shows that expression of truncation mutants of either TWIST1 or RELA prevents most binding between TWIST1 and RELA. Co-expression of both truncation mutants further reduces binding, validating the truncated domains as required binding sites for their counterpart proteins. RELA $\triangle 307$ bands have been shown separate from WT due to difference in electrophoretic mobility on account of reduced size. c Quantitation of duplicate western blots following CoIP of TWIST1 with indicated RELA alleles. $\triangle 307$ mutation reduced protein binding by $90 \%$ on average. Graphed is the ratio of TWIST1 to RELA, each normalized to their respective inputs. $\mathbf{d}$ Dual luciferase assay reveals that while the $\Delta 307$ allele of RELA reduces TWIST1-mediated upregulation of IL-8 when compared to WT RELA, the same trend is seen in the absence of TWIST1. This suggests that the C-terminal portion of RELA is required not only for TWIST1 binding, but also for proper transcriptional activity. Graph represents firefly luciferase expression normalized to renilla luciferase for each condition. Error bars represent standard deviations of biological triplicate experiments. WT TWIST1 + WT RELA condition was used as the basis for statistical comparisons. pGL3 lacking the IL-8 promoter was used as a negative control. ${ }^{* * *}, p<.001 ;{ }^{* * * *}, p<.0001$

dependent reduction in FFluc expression (Fig. 4e). Thus, the TWIST1-driven IL-8 pathway can be inhibited by direct competition using the WR domain.

\section{GFP-WR fusion protein leads to TWIST1 degradation}

As GFP-WR was primarily expressed in the cytoplasm of transfected cells (Additional file 1: Figure S2), we hypothesized that GFP-WR was sequestering TWIST1 in the cytoplasm. In order to test this hypothesis, we isolated cytoplasmic and nuclear cell fractions and analyzed the levels of TWIST1 found in each. Western blot of fractionated cells showed that in both cytoplasmic and nuclear fractions, the protein levels of TWIST1 and GFP decreased as the proportion of GFP-WR transfected was 
a

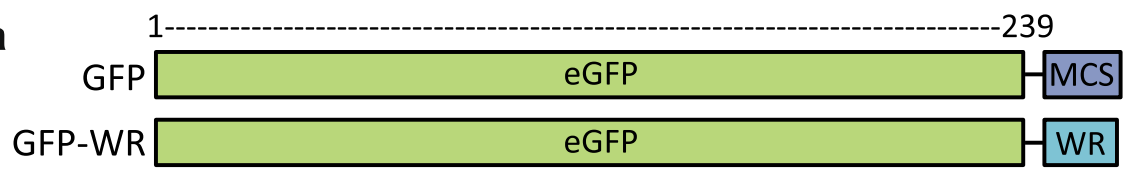

b

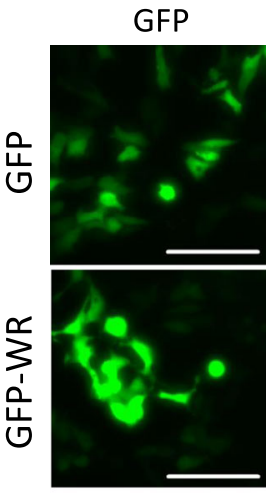

Phase

Merge
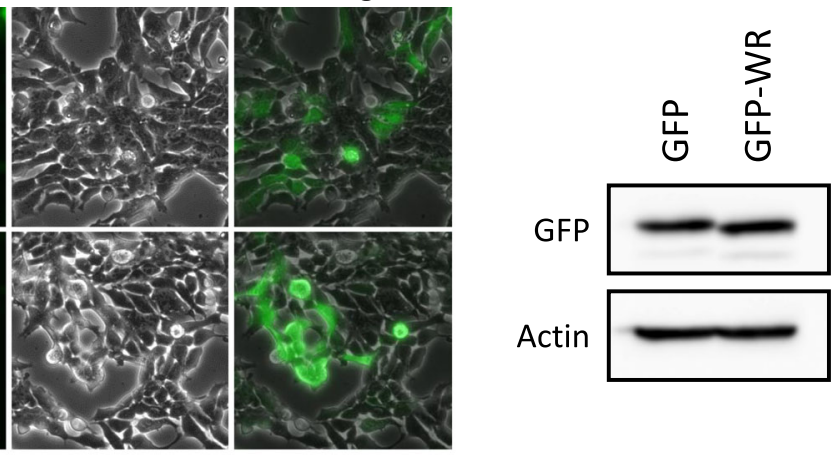

C

d
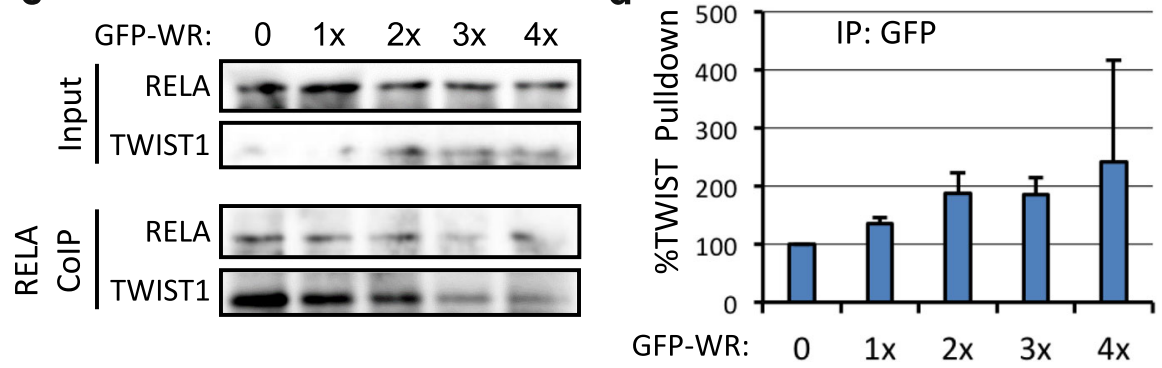

e

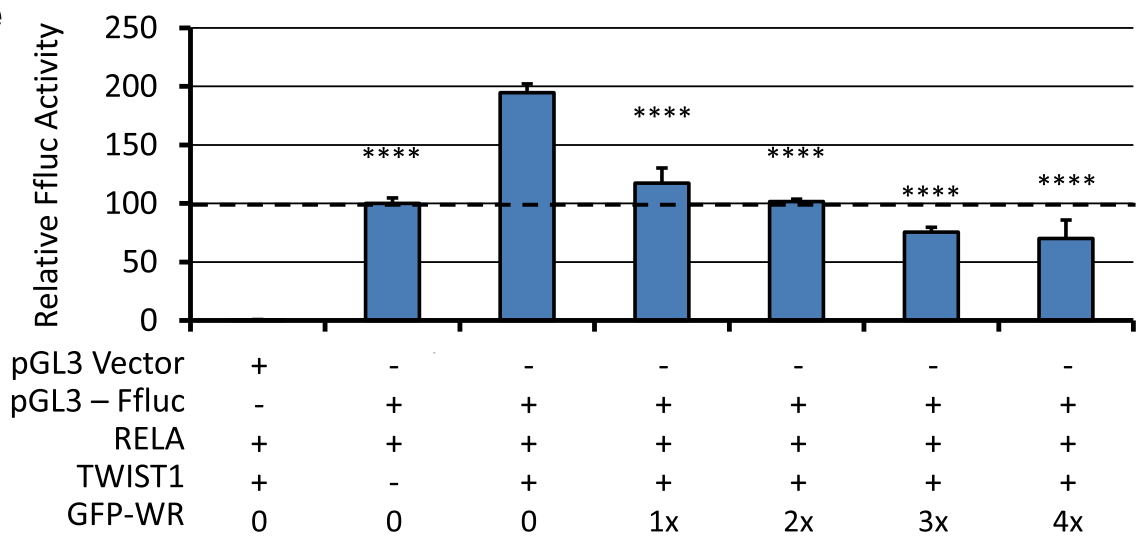

Fig. 4 Competitive inhibition of TWIST1 WR domain binding. a Schematic representation of GFP alleles used. GFP contains 23 amino acids encoded by the multiple cloning site of the vector at its C-terminus. GFP-WR contains the first two such amino acids (Leu-Glu encoded by Xhol restriction site), followed by the 20 amino acids of the WR domain. Thus, the two alleles have indistinguishable molecular weights. $\mathbf{b}$ Left, fluorescent microscopy shows that GFP and GFP-WR are expressed at similar levels and in similar patterns in HEK-293 cells. Scale bar, 100 um. Right, Western blot confirms equal GFP and GFP-WR expression. c CoIP with RELA pulldown reveals that in the presence of increasing GFP-WR expression, TWIST1-RELA binding is reduced in a dose-dependent manner. $\mathbf{d}$ CoIP with GFP pulldown reveals that increasing GFP-WR dose results in more TWIST1 co-precipitated with GFP. Graph represents ratio of TWIST1 to GFP, normalized to their respective inputs. Error bars, standard deviation of duplicate experiments. e Dual luciferase assay demonstrates that as seen in the RELA CoIP, there is a dose dependent drop in IL-8 driven luciferase expression with increasing dose of GFP-WR inhibitor. Graph represents firefly luciferase expression normalized to renilla luciferase for each condition. Error bars represent standard deviation of biological triplicate experiments. GFP without GFP-WR condition was used as the basis for statistical comparisons. pGL3 lacking the IL-8 promoter was used as a negative control. ${ }^{* * *}, p<.0001$ 
increased (Fig. 5a). This suggested that rather than sequestration, the interactions between these proteins may lead to their degradation, as seen previously following altered binding of TWIST1 to partner proteins [19, 22]. In order to test this hypothesis, we transfected HEK-293 cells with TWIST1, RELA, and either GFP or GFP-WR and after $24 \mathrm{~h}$, treated with cycloheximide (CHX) to prevent further protein production. TWIST1 was degraded more quickly in cells expressing GFP-WR than in those expressing GFP (Fig. 5b), suggesting that GFP-WR leads to TWIST1 turnover. To determine if this process was dependent on proteasomal activity, we transefcted HEK293 cells and treated them with the proteasome inhibitor MG132 overnight. Western blots show that MG132 was able to increase the levels of TWIST1 and GFP by up to two fold in the cytoplasmic fraction of these cells (Fig. 5c). Finally, in order to determine the effect of proteasome inhibition on IL-8 promoter activity, a dual luciferase assay was once again employed. Treatment with MG132 following GFP-WR expression increased IL-8 promoter activity two fold, correlating with increased TWIST1 expression observed following MG132 treatment (Fig. 5d).

\section{Discussion}

We and others have shown that the TWIST1 WR domain is important for TWIST1 protein binding and transcription factor activities, and here we have analyzed further the specific interaction between TWIST1 and RELA. We demonstrated previously that the WR domain was required for the formation of a complex between these two proteins, but that TWIST1-DNA binding was dispensable [18]. We further showed that the production of IL- 8 was reduced by loss of binding as a result of deleting the WR domain [18]. In the present study, we identified three highly conserved residues within the WR domain and mutated each to alanine in order to ascertain their role in TWIST1 activity. We observed that all three mutations led to a $50 \%$ reduction in TWIST1-RELA co-precipitation and downstream IL-8 promoter activity; the triple mutant further reduced RELA binding and IL-8 promoter activity. These findings suggest that the central region of the WR domain (W190, R191, E193) is important for protein-protein interactions involving TWIST1. This function may explain their evolutionary sequence conservation.

It is important to note that the data presented here cannot preclude the existence of additional or intermediary protein members of the TWIST1-RELA complex, although their overexpression in HEK-293 cells in the absence of other exogenous cofactors and previous work on these two proteins suggests that a direct binding interaction is likely [23].
Further studies, including structural biology approaches, will be necessary to fully elucidate the TWIST1-RELA binding interaction. No crystal structure for full length TWIST1 presently exists. However, a computational model predicts a helical structure for much of the WR domain and also suggests an interface that binds to p53 [19]. The R191 residue in particular was responsible for disrupting p53 post-translational modifications, leading to p53 degradation [19]. We have shown here that the WR domain interacts with a RELA transactivation domain downstream of the REL homology domain, which also has yet to be structurally characterized. Other groups have shown also that the WR-domain of TWIST1 binds to Sox10 and Runx3 [24, 25], and additional binding partners may yet be identified. Further studies are needed to recognize structural motifs that may predict TWIST1binding sites on additional cellular proteins.

Having shown that the bHLH domain of TWIST1 was not required for IL-8 regulation [18], we hypothesized that separation of function would be possible, and that we could independently study the DNA binding and protein binding functions of TWIST1. However, Gajula et al. showed that TWIST1 lacking the WR domain was unable to promote metastasis in an in vivo model of prostate cancer. Specifically, they found that TWIST1mediated regulation of Hoxa9 at the transcriptional level was responsible for the phenotype they observed [20]. A possible explanation for this finding is that TWIST1responsive promoters can contain tandem E-box sequences. Both E-boxes are bound by TWIST1 heterodimers, which then interact via their WR domains to form a transient tetramer [17]. Thus, whether directly bound to DNA or bound to protein cofactors, there is now strong evidence that WR domain interactions lie at the heart of many TWIST1 signaling processes.

Targeting of the WR domain offers a potential therapeutic approach to simultaneously disrupt protein binding, transcription factor activities, and rate of recycling of the TWIST1 protein. To test this hypothesis, a GFP fusion protein including the WR domain was created and used to inhibit normal TWIST1-RELA complex formation and IL-8 promoter regulation. The GFP-WR fusion protein successfully reduced TWIST1 activity, and led to TWIST1 degradation in a dose dependent manner. The finding that TWIST1 was co-precipitated with GFP-WR suggests that these proteins are interacting via their WR domains, blocking WR domain binding to other partner proteins.

Importantly, TWIST1 inhibition via blocking of binding and subsequent degradation has a natural analogue, supporting its efficacy: TWIST1 is known to be sequestered by HLH inhibitor of DNA binding (Id) family members 2 and 4, preventing its binding to other partners $[26,27]$. Moreover, mutations in TWIST1 found in 
a

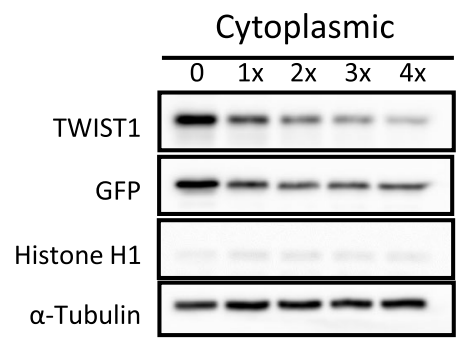

b

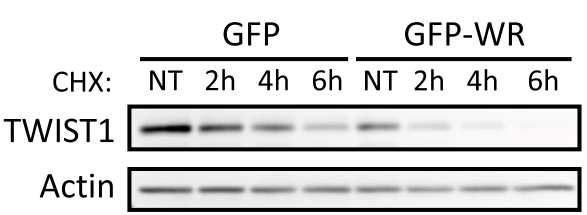

C

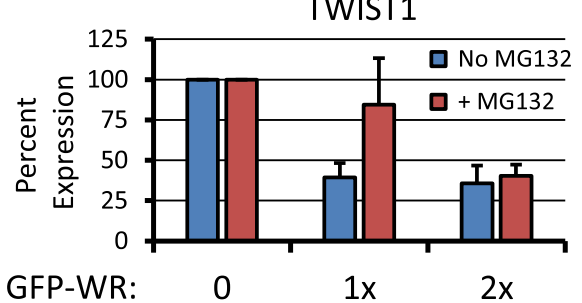

Nuclear
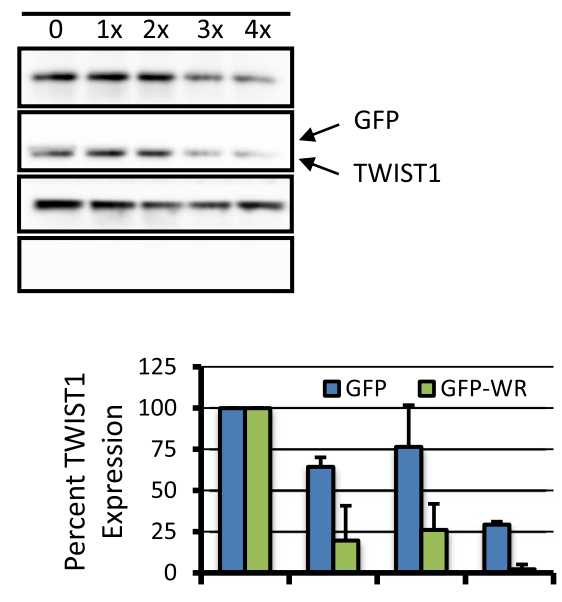

CHX: NT $2 \mathrm{~h} \quad 4 \mathrm{~h} \quad 6 \mathrm{~h}$

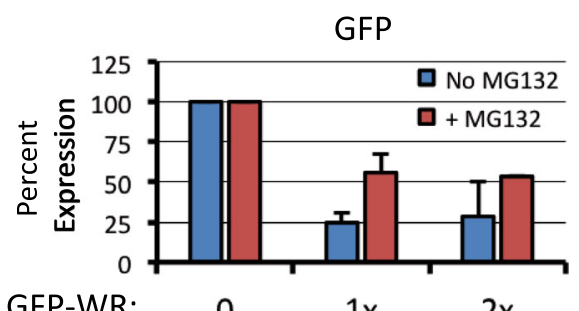

d

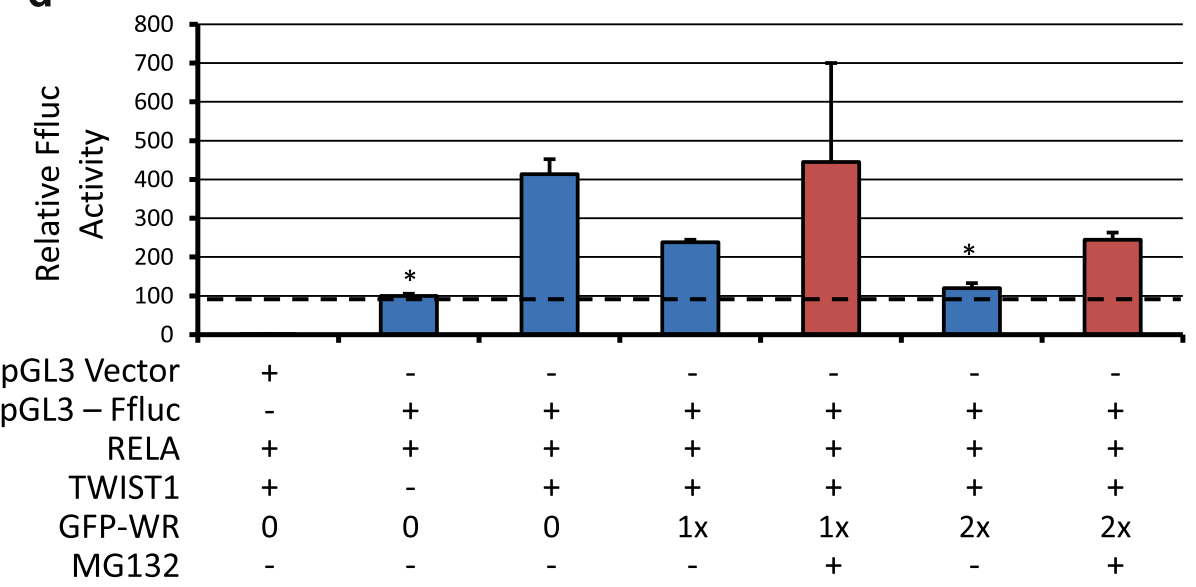

Fig. 5 Mechanism of GFP-WR action. a Fractionation experiments reveal an overall decrease in TWIST1 and GFP protein expression in the cytoplasm as the level of GFP-WR co-expressed in cells increases. TWIST levels also decrease in the nucleus, but GFP-WR is not expressed in the nuclear fraction. This suggests that GFP-WR expression may lead to TWIST1 degradation. Histone H1 and alpha tubulin were used as nuclear and cytoplasmic markers, respectively. b Cycloheximide (CHX) treatment of cells co-transfected with TWIST1 and either GFP or GFP-WR. Left, representative western blot demonstrates more rapid turnover of TWIST1 in the presence of GFP-WR than GFP. Right, quantitation of duplicate experiments. c TWIST1 and GFP levels in the cytoplasmic fraction show a 2-fold increase upon MG132 treatment at 1x dose of GFP-WR (biological duplicate experiments, each condition normalized to its 0 GFP-WR control). d Dual luciferase assay demonstrates that MG132 treatment increases IL-8 driven FFluc expression. Graph represents firefly luciferase expression normalized to renilla luciferase for each condition. Error bars represent standard deviations of biological triplicate experiments. GFP without GFP-WR or MG132 was used as the basis for statistical comparisons. pGL3 lacking the IL-8 promoter was used as a negative control. Error bars, standard deviation. ${ }^{*}, p<.05$ 
Saethre-Chotzen Syndrome that prevent its dimerization and nuclear translocation have been shown to lead to degradation of the protein [22].

Future work will focus on further characterization of TWIST1 turnover and GFP-WR mechanism. It is possible that binding between the WR domains of TWIST1 and GFP-WR alone is sufficient to lead to TWIST1 degradation, but it is also possible that the GFP component of the fusion protein contributes to TWIST1 inhibition. For instance, the size of the protein may block other TWIST1 binding partners from binding, or prevent post-translational modifications of TWIST1 required for its stability or activity. To assess the efficacy of the WR domain alone, we have created a WR domain peptide fused to a nona-arginine cell penetrating leader sequence, and will test its ability to inhibit TWIST1 binding and activity. Use of this peptide design is supported by the efficacy of a similar peptide mimic of BRCA1-IRIS, which led to degradation of IRIS and reversal of its pro-drug resistance effects [28].

Future work will also focus on additional therapeutics against TWIST1. We have already demonstrated the efficacy of siRNA against TWIST1 delivered using multiple nanoparticle platforms. Dendrimers carrying siRNA against TWIST1 reduced migration in vitro and homed to tumors in a xenograft model of triple negative breast cancer in mice [29]. In addition, mesoporous silica particles carrying chemically modified siRNA reduced tumor size in an in vivo melanoma model via reduction of angiogenesis [30] and reversed cisplatin resistance in an ovarian cancer model, leading to reduction of tumor growth in mice [31]. We have also shown that cells exhibited reduced Akt signaling and in vivo survival in response to cisplatin treatment when TWIST1 expression was reduced by shRNA [15].

TWIST1 is an attractive target for novel therapies: it is rarely expressed in adult tissues, reducing the chance of off-site effects [32], and it plays a role in multiple cancer processes correlated with poor outcome, such as metastatic spread, angiogenesis, resistance to apoptosis, drug resistance pathways, and cancer cell stemness (Fig. 1a) $[7,9,11,14,33,34]$. Further development of multiple approaches to TWIST1 targeting is warranted, as patients at the highest risk, and who therefore tend to have the fewest therapeutic options, may be in a position to benefit most from TWIST1-targeted treatment.

\section{Conclusions}

We have shown here that the formation of a TWIST1RELA complex is partially dependent on the well conserved W190, R191, and E193 residues of the TWIST1 WR domain, and that mutations at these positions reduce binding and downstream IL-8 promoter activation. These effects may be the result of lower binding affinity due to changes to the binding interface or to destabilization of the WR domain fold. We have further showed that the C-terminus of RELA is involved both in TWIST1 binding and IL-8 promoter activation. Finally, we have demonstrated a competitive inhibitor of the TWIST1 WR domain has therapeutic potential, leading to the degradation of TWIST1 protein. Further investigations of WR-mediated binding interactions and development of TWIST1-targeted therapies may be of great value to patients suffering from advanced, drug resistant carcinomas.

\section{Additional file}

Additional file 1: Figure S1. TWIST1 mutation decreases IL-8 promoter activity in Ovcar3 cells. Replicating the experiment shown for Ovcar4 in Fig. 2 shows a similar trend in the Ovcar3 cell line. As Ovcar3 showed a smaller degree of induction with TWIST1 expression, Ovcar4 was used as the cell line of choice for remaining functional assays. Figure S2. Confocal images of GFP allele localization. GFP lacking the WR domain is visible throughout the cell, including the nucleus, as indicated by DAPI staining. GFP-WR signal appears distinct from DAPI-positive nuclei. (PDF 456 kb)

\section{Abbreviations}

CHX: Cycloheximide; CoIP: Co-immunoprecipitation; CSC: Cancer stem cell; EMT: Epithelial to mesenchymal transition; FBS: Fetal bovine serum; FFluc: Firefly luciferase; GFP: Green fluorescent protein; GFP-WR: GFP-WR domain fusion protein; Id: Inhibitor of DNA binding; IL-8: Interleukin 8; PBS: Phosphate buffered saline; PBST: PBS with 0.1\% Tween-20

\section{Acknowledgements}

The authors wish to thank Abby Chiang, Leslie Cisneros, and Emily Ye for their assistance with experiments presented herein. Sophia also assisted in the preparation of the manuscript, as did Dr. Virginia Hetrick and Evan Finlay. We also wish to thank Dr. Ren-Jang Lin for his suggestions regarding GFP-WR mechanism of action. We thank Dr. Gil Mor of the Yale University School of Medicine for sharing his time and resources. We thank Loren Quintanar and the City of Hope Light Microscopy Core for their assistance with confocal microscopy. Research reported in this publication included work performed in the City of Hope Integrated Genomics Core supported by the National Cancer Institute of the National Institutes of Health under award number P30CA33572. The content is solely the responsibility of the authors and does not necessarily represent the official views of the National Institutes of Health. We thank the core for sequencing help on the project.

\section{Funding}

Funding for this work came from an $\mathrm{NIH} \mathrm{NCI} \mathrm{Excellence} \mathrm{Award} \mathrm{and} \mathrm{City} \mathrm{of}$ Hope Women's Cancer Program Pilot Grant.

\section{Availability of data and materials}

All data supporting the conclusions drawn herein are presented in the figures shown.

\section{Authors' contributions}

CR designed research, conducted assays, and wrote the paper. SS performed confocal microscopy. JL assisted with assays and created mutant alleles. TD, JW, and CG conceived of the study, directed research, and contributed to writing the paper. All authors read and approved the manuscript.

Competing interests

The authors declare they have no competing interests.

Consent for publication

Not applicable.

Ethics approval and consent to participate

Not applicable, no human subjects were used in this study. 


\section{Publishers Note}

Springer Nature remains neutral with regard to jurisdictional claims in published maps and institutional affiliations.

\section{Author details Parnassus Ave, San Francisco, CA 94143, USA. \\ Received: 10 March 2016 Accepted: 4 March 2017 Published online: 10 March 2017}

${ }^{1}$ City of Hope, 1500 E Duarte Rd, Duarte, CA 91010, USA. ${ }^{2}$ California State University, 5151 State University Drive, Los Angeles, CA 90032, USA. ${ }^{3}$ Present address: Yale University School of Medicine, 333 Cedar Street, New Haven, CT 06520, USA. ${ }^{4}$ Present address: University of California, San Francisco, 505

\section{References}

1. Nguyen DX, Bos PD, Massague J. Metastasis: from dissemination to organspecific colonization. Nat Rev Cancer. 2009;9(4):274-84.

2. Kim EY, Cho EN, Park HS, Kim A, Hong JY, Lim S, Youn JP, Hwang SY, Chang YS. Genetic heterogeneity of actionable genes between primary and metastatic tumor in lung adenocarcinoma. BMC Cancer. 2016;16:27.

3. Trimble EL, Wright J, Christian MC. Treatment of platinum-resistant ovarian cancer. Expert Opin Pharmacother. 2001;2(8):1299-306.

4. van Jaarsveld MT, Helleman J, Boersma AW, van Kuijk PF, van ljcken WF, Despierre E, Vergote I, Mathijssen RH, Berns EM, Verweij J, et al. miR-141 regulates KEAP1 and modulates cisplatin sensitivity in ovarian cancer cells. Oncogene. 2013;32(36):4284-93.

5. Ferlay J, Parkin DM, Steliarova-Foucher E. Estimates of cancer incidence and mortality in Europe in 2008. Eur J Cancer. 2010;46(4):765-81.

6. Simpson P. Maternal-zygotic gene interactions during formation of the dorsoventral pattern in drosophila embryos. Genetics. 1983;105(3):615-32.

7. Yang J, Mani SA, Donaher JL, Ramaswamy S, Itzykson RA, Come C, Savagner P, Gitelman I, Richardson A, Weinberg RA. Twist, a master regulator of morphogenesis, plays an essential role in tumor metastasis. Cell. 2004;117(7):927-39.

8. Fu J, Qin L, He T, Qin J, Hong J, Wong J, Liao L, Xu J. The TWIST/Mi2/NuRD protein complex and its essential role in cancer metastasis. Cell Res. 2011;21(2):275-89.

9. Low-Marchelli JM, Ardi VC, Vizcarra EA, van Rooijen N, Quigley JP, Yang J. Twist1 induces CCL2 and recruits macrophages to promote angiogenesis. Cancer Res. 2013;73(2):662-71.

10. Kong D, Li Y, Wang Z, Sarkar FH. Cancer stem cells and epithelial-tomesenchymal transition (emt)-phenotypic cells: are they cousins or twins? Cancers. 2011;3(1):716-29.

11. Vesuna F, Lisok A, Kimble B, Raman V. Twist modulates breast cancer stem cells by transcriptional regulation of CD24 expression. Neoplasia (New York, NY). 2009;11(12):1318-28.

12. Li W, Wang Z, Zha L, Kong D, Liao G, Li H: HMGA2 regulates epithelialmesenchymal transition and the acquisition of tumor stem cell properties through TWIST1 in gastric cancer. Oncol Rep 2016.

13. Uthaya Kumar DB, Chen CL, Liu JC, Feldman DE, Sher LS, French S, DiNorcia J, French SW, Naini BV, Junrungsee $S$, et al. TLR4 signaling via NANOG cooperates with STAT3 to activate Twist1 and promote formation of tumorinitiating stem-like cells in livers of mice. Gastroenterology. 2016;150(3):707-19.

14. Bridges RS, Kass D, Loh K, Glackin C, Borczuk AC, Greenberg S. Gene expression profiling of pulmonary fibrosis identifies Twist1 as an antiapoptotic molecular "rectifier" of growth factor signaling. Am J Pathol. 2009;175(6):2351-61.

15. Roberts CM, Tran MA, Pitruzzello MC, Wen W, Loeza J, Dellinger TH, Mor G, Glackin CA. TWIST1 drives cisplatin resistance and cell survival in an ovarian cancer model, via upregulation of GAS6, L1CAM, and Akt signalling. Sci Rep. 2016;6:37652

16. Murray SS, Glackin CA, Winters KA, Gazit D, Kahn AJ, Murray EJ. Expression of helix-loop-helix regulatory genes during differentiation of mouse osteoblastic cells. J Bone Miner Res. 1992;7(10):1131-8.

17. Chang AT, Liu Y, Ayyanathan K, Benner C, Jiang Y, Prokop JW, Paz H, Wang D, Li HR, Fu XD, et al. An evolutionarily conserved DNA architecture determines target specificity of the TWIST family bHLH transcription factors. Genes Dev. 2015;29(6):603-16.

18. Li S, Kendall SE, Raices R, Finlay J, Covarrubias M, Liu Z, Lowe G, Lin YH, Teh YH, Leigh V, et al. TWIST1 associates with NF-kappaB subunit RELA via carboxyl-terminal WR domain to promote cell autonomous invasion through IL8 production. BMC Biol. 2012;10:73.

19. Piccinin S, Tonin E, Sessa S, Demontis S, Rossi S, Pecciarini L, Zanatta L, Pivetta F, Grizzo A, Sonego M, et al. A "twist box" code of p53 inactivation: twist box: p53 interaction promotes p53 degradation. Cancer Cell. 2012;22(3):404-15.

20. Gajula RP, Chettiar ST, Williams RD, Thiyagarajan S, Kato Y, Aziz K, Wang $R$, Gandhi N, Wild AT, Vesuna F, et al. The twist box domain is required for Twist1-induced prostate cancer metastasis. Mol Cancer Res. 2013;11(11):1387-400

21. Chen YQ, Ghosh S, Ghosh G. A novel DNA recognition mode by the NFkappa B p65 homodimer. Nat Struct Biol. 1998;5(1):67-73.

22. El Ghouzzi V, Legeai-Mallet L, Aresta S, Benoist C, Munnich A, de Gunzburg J, Bonaventure J. Saethre-Chotzen mutations cause TWIST protein degradation or impaired nuclear location. Hum Mol Genet. 2000;9(5):813-9.

23. Sosic D, Richardson JA, Yu K, Ornitz DM, Olson EN. Twist regulates cytokine gene expression through a negative feedback loop that represses NFkappaB activity. Cell. 2003;112(2):169-80.

24. Vincentz JW, Firulli BA, Lin A, Spicer DB, Howard MJ, Firulli AB. Twist1 controls a cell-specification switch governing cell fate decisions within the cardiac neural crest. PLoS Genet. 2013;9(3):e1003405.

25. Pham DVJ, Firulli AB, Kaplan MH. Twist1 regulates Ifng expression in Th1 cells by interfering with Runx3 function. J Immunol. 2012;189(2):832-40.

26. Yang J, Velikoff M, Agarwal M, Disayabutr S, Wolters PJ, Kim KK. Overexpression of inhibitor of DNA-binding 2 attenuates pulmonary fibrosis through regulation of c-Abl and Twist. Am J Pathol. 2015;185(4):1001-11.

27. Rahme GJ, Israel MA. Id4 suppresses MMP2-mediated invasion of glioblastoma-derived cells by direct inactivation of Twist1 function. Oncogene. 2015;34(1):53-62.

28. Paul BT, Blanchard Z, Ridgway M, EIShamy WM. BRCA1-IRIS inactivation sensitizes ovarian tumors to cisplatin. Oncogene. 2015;34(23):3036-52.

29. Finlay J, Roberts CM, Lowe G, Loeza J, Rossi JJ, Glackin CA. RNA-based TWIST1 inhibition via dendrimer complex to reduce breast cancer cell metastasis. Biomed Res Int. 2015;2015:382745.

30. Finlay J, Roberts CM, Dong J, Zink JI, Tamanoi F, Glackin CA. Mesoporous silica nanoparticle delivery of chemically modified siRNA against TWIST1 leads to reduced tumor burden. Nanomedicine. 2015;11(7):1657-66.

31. Roberts CM, Shahin SA, Wen W, Finlay JB, Dong J, Wang R, Dellinger TH, Zink J, Tamanoi F, Glackin CA: Nanoparticle delivery of siRNA against TWIST to reduce drug resistance and tumor growth in ovarian cancer models. Nanomedicine: nanotechnology, biology, and medicine 2016.

32. Wang SMCV, Pignolo RJ, Rotenberg MO, Cristofalo VJ, Sierra F. Cloning of the human twist gene: its expression is retained in adult mesodermallyderived tissues. Gene. 1997;187(1):83-92.

33. Haslehurst AM, Koti M, Dharsee M, Nuin P, Evans K, Geraci J, Childs T, Chen J, Li J, Weberpals J, et al. EMT transcription factors snail and slug directly contribute to cisplatin resistance in ovarian cancer. BMC Cancer. 2012;12:91.

34. Wang Y, Qu Y, Niu XL, Sun WJ, Zhang XL, Li LZ. Autocrine production of interleukin-8 confers cisplatin and paclitaxel resistance in ovarian cancer cells. Cytokine. 2011;56(2):365-75.

\section{Submit your next manuscript to BioMed Central and we will help you at every step:}

- We accept pre-submission inquiries

- Our selector tool helps you to find the most relevant journal

- We provide round the clock customer support

- Convenient online submission

- Thorough peer review

- Inclusion in PubMed and all major indexing services

- Maximum visibility for your research

Submit your manuscript at www.biomedcentral.com/submit
Biomed Central 